\title{
Prevalence and Antibiotic Susceptibility Patterns of Methicillin Resistant Staphylococcus Aureus in Patients Attending the Laquintinie Hospital Douala, Cameroon
}

\author{
Marie Ebob Agbortabot Bissong 2, *, Therese Wirgham", Mbi Alice Enekegbe ${ }^{1}$, \\ Peter Thelma Ngwa Niba ${ }^{1}$, Frank Eric Tatsing Foka ${ }^{3}$ \\ ${ }^{1}$ Department of Medical Laboratory Sciences, St. Louis University Institute of Health and Biomedical Sciences, Bamenda, Cameroon \\ ${ }^{2}$ Department of Biomedical Sciences, Faculty of Health Sciences, University of Bamenda, Bambili, Cameroon \\ ${ }^{3}$ Department of Biological Sciences, Faculty of Agriculture, Science and Technology, North West University, Mafikeng, South Africa
}

Email address:

mabissong@yahoo.com (M. E. A. Bissong), thercyvisa@yahoo.com (T. Wirgham), enekegbe2000@yahoo.com (M. A. Enekegbe), thelma2009@yahoo.co.uk (P. T. N. Niba), frankeric2023@yahoo.fr (F. E. T. Foka)

${ }^{*}$ Corresponding author

\section{To cite this article:}

Marie Ebob Agbortabot Bissong, Therese Wirgham, Mbi Alice Enekegbe, Peter Thelma Ngwa Niba, Frank Eric Tatsing Foka. Prevalence and Antibiotic Susceptibility Patterns of Methicillin Resistant Staphylococcus Aureus in Patients Attending the Laquintinie Hospital Douala, Cameroon. European Journal of Clinical and Biomedical Sciences. Vol. 2, No. 6, 2016, pp. 92-96. doi: 10.11648/j.ejcbs.20160206.16

Received: October 31, 2016; Accepted: December 3, 2016; Published: January 5, 2017

\begin{abstract}
Methicillin Resistant Staphylococcus aureus (MRSA) is a major health problem worldwide. Diseases caused by these microorganisms most often are difficult to treat consequently, present with severe outcomes. Despite the increase association of MRSA in most hospital acquired infections, surveillance systems to control the spread of these infections are lacking especially in the developing countries. The present study is part of a major study developed to trail the incidence of multidrug resistant pathogens in clinical settings using available resources, with the aim to gather relevant data for the management and control of nosocomial infections. The specific objective of the current study is therefore to determine the prevalence and antimicrobial susceptibility of MRSA isolates from one of the largest hospitals in the country. This was a hospital based cross sectional study involving 114 participants, conducted between March and June 2016. Various clinical specimens were inoculated on to blood agar and mannitol salt agar and incubated at $35-37^{\circ} \mathrm{C}$ aerobically for $18-24$ hours for the isolation of $S$. aureus. Isolates were identified using the catalase and coagulase tests. Sensitivity testing was done using the Kirby Bauer disk diffusion method. Data were analysed using SPSS version 20.0. Out of 114 samples analysed, S. aureus was isolated from $33(28.95 \%)$ while MRSA was detected in $15(13.16 \%)$. Antimicrobial susceptibility testing revealed high resistance (80-100\%) to penicillins and no resistance to amikacin, lincomycin and ofloxacin. Multidrug resistance to at least three antibiotics was also observed among MRSA isolates. MRSA and multidrug resistant $S$. aureus are common in the study site. Therefore, there is a need for the improvement of surveillance systems to monitor and curb the spread of these resistant pathogens.
\end{abstract}

Keywords: Prevalence, Antimicrobial Resistance, Methicillin Resistant Staphylococcus Aureus, Laquintinie Hospital, Cameroon

\section{Introduction}

Methicillin resistant Staphylococcus aureus (MRSA) has been reported as a common cause of hospital and community acquired infections worldwide [1, 2]. Infections caused by these microorganisms present with severe outcomes and often involves prolonged hospital stay, long-term disability, increased resistance of microorganisms to antimicrobials, additional financial burden for health systems, high costs for patients and their family, and unnecessary deaths [3]. MRSA strains exhibit resistance to oxacillin or methicillin and other antimicrobial agents and this has been used in the diagnosis of these strains $[4,5]$. The emergence of resistance in these strains of $S$. aureus is mediated by a change in penicillin 
binding protein (PBP2a) which is encoded by the Mec A gene, resulting to strains with multiple resistance to various antibiotics $[1,6]$.

Staphylococcus aureus has been previously implicated in different infections including genital, skin, bone, ear, urinary tract, and post-operation wounds [2, 7, 8]. The increasing prevalence of MRSA multiple-drug resistant strains is a limitation to the treatment and management of MRSA associated infections. Consequently, MRSA are increasingly being recognised as major public health threat both in the developed and developing setups. In spite of the availability of a diversity of antibiotics for the treatment of staphylococcal infections, the prevalence of MRSAassociated infections remains on the increase. According to National Nosocomial Infection Surveillance in USA, the occurrence of MRSA causing nosocomial infection has increased from about $2.0 \%$ in 1974 to $22.0 \%$ in 1995 and then to $63.0 \%$ in 2004 [9].

The World Health Organization (WHO) recommends continuous surveillance as a means of controlling the spread of diseases caused by resistant pathogens [3]. Such systems of tracking diseases are lacking in most developing countries. In the absence of such surveillance systems, data from prevalence studies may be helpful. In Cameroon, there has been an increase in the prevalence of MSRA from $21.3 \%$ in $2003,13.8 \%$ in 2013 and $72 \%$ in 2015 [7, 10, 11]. As such there is a need to track the occurrence of these pathogens. The present study is part of a major study which is focused on monitoring the incidence of multidrug resistant pathogens in clinical settings with the aim to gather relevant data for the management and control of nosocomial infections. The specific objective of the current study is therefore to determine the prevalence and antimicrobial susceptibility of MRSA isolates from one of the largest hospitals in the country.

\section{Materials and Methods}

\subsection{Study Location}

The present study was a hospital-based cross-sectional study conducted on both in and out patients attending the Laquintinie Hospital from March to June, 2016. This hospital is a referral hospital located in the economic capital of the Nation and as such, it serves patients from all over the country.

\subsection{Ethical Considerations}

Ethical clearance for this study was obtained from the Littoral Regional delegation for Public Health. All participants were issued consent forms and only those who consented were enrolled into the study.

\subsection{Specimen Collection}

A total of 114 consented participants who presented with varied skin and soft tissue infections were recruited into the study. Specimens such as pus, ear discharges, urine, genital and throat swabs were collected aseptically and transported at $+4^{\circ} \mathrm{C}$ to the Microbiology laboratory within two hours of collection for analysis.

\subsection{Laboratory Analyses}

\subsubsection{Isolation and Identification of S. aureus}

Each sample was inoculated unto mannitol salt agar (MSA) and blood agar (BA) and the plates were incubated at $37^{\circ} \mathrm{C}$ for 24 hours [5]. Plates were observed for characteristic yellow colonies on MSA and beta $(\beta)$ haemolysis on BA. Isolates were Gram stained and identified using the catalase and coagulase tests. Methicillin resistance was assessed by determining the resistance profile of $S$. aureus isolates to oxacillin [5].

\subsubsection{Antibiotic Sensitivity Testing and Interpretation}

The antimicrobial susceptibility testing of all $S$. aureus isolates to different antibiotics were carried out according to the guidelines of Clinical and Laboratory Standards Institute (CLSI) by Kirby-Bauer disc diffusion method [12]. The 10 antibiotics tested were oxacillin $(\mathrm{OX}-1 \mu \mathrm{g})$, penicillin $(\mathrm{P}-10$

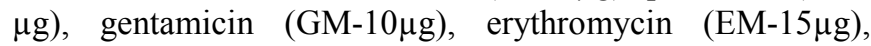
amoxicillin-clavulanic acid (AMC-30 $\mu \mathrm{g}$ ), lincomycin (LN-

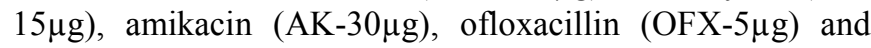
vancomycin $(\mathrm{VA}-30 \mu \mathrm{g})$ (Liofilchem, Italy).

\subsection{Statistical Analysis}

Data were analysed using the International Business Machine Statistical software Package for Social Sciences (IBM SPSS) version 20.0. Significant differences between categorical variables were determined using the Chi-Square test at a 95\% confidence level. $\mathrm{P}<0.05$ was considered statistically significant.

\section{Results}

\subsection{Prevalence of MRSA}

Out of 114 clinical samples analysed, S. aureus was isolated from 33 (28.95\%) and MRSA from 15 (13.16\%); and of the 33 S. aureus isolates, $45.45 \%$ were MRSA (Figure 1).

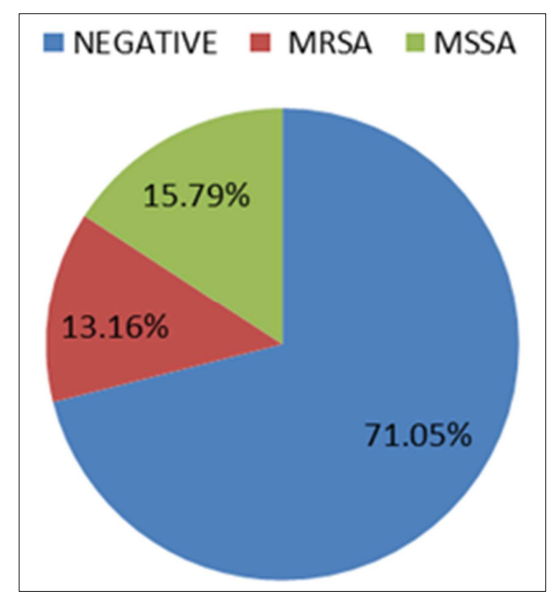

Figure 1. Prevalence of Staphylococcus aureus. 


\subsection{Antimicrobial Resistance}

The results of antimicrobial susceptibility pattern of MRSA isolates are shown on Figure 2. All the isolates were resistant to oxacillin. Of the 15 isolates, $12(80 \%)$ were resistant to penicillin and $10(66.7 \%)$ were resistant to erythromycin. Isolates showed no resistance to lincomycin, amikacin and ofloxacin. One isolate $(6.7 \%)$ showed resistance to vancomycin.

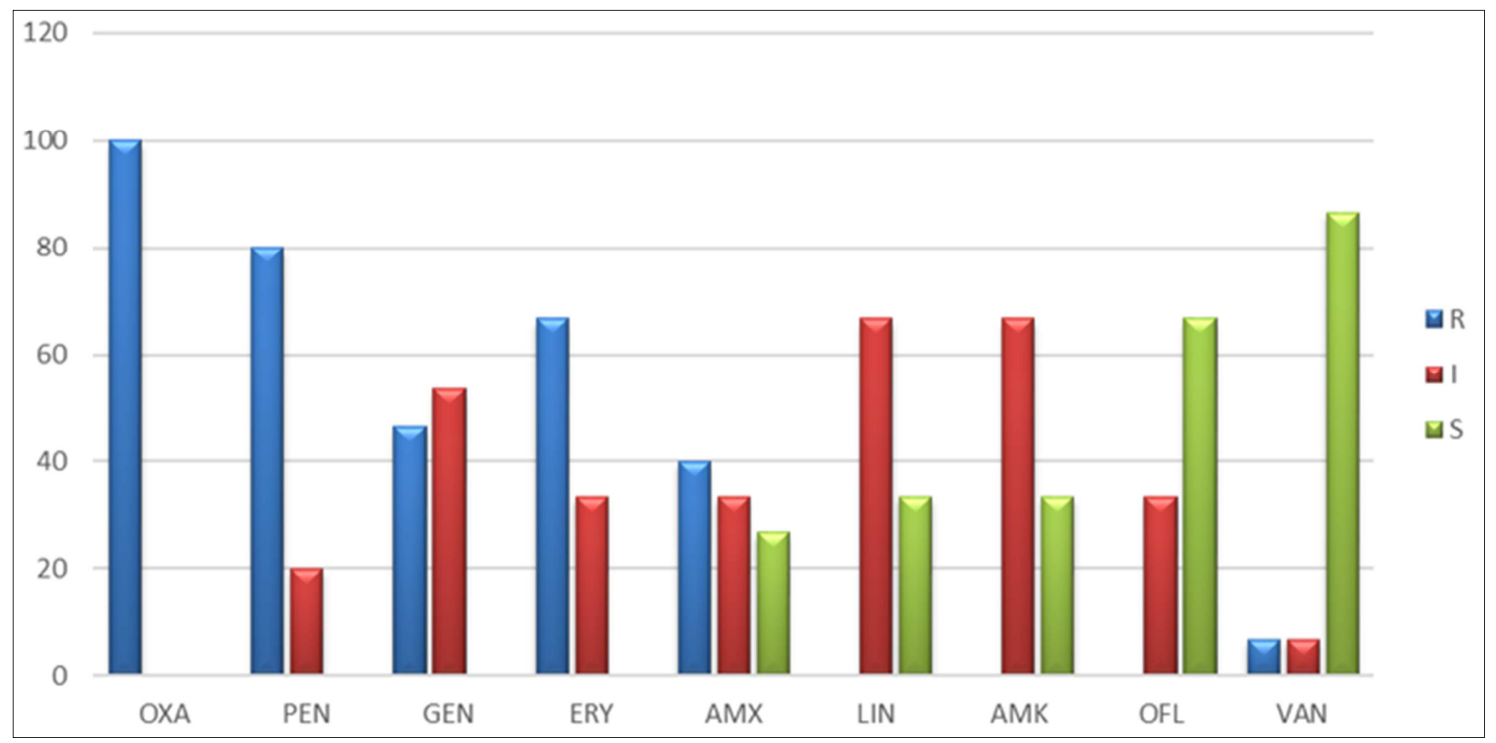

Figure 2. Antimicrobial susceptibility pattern of MRSA isolates.

$\mathrm{R}=$ Resistance, $\mathrm{I}=$ Intermediate resistance, $\mathrm{S}=$ Sensitive, OXA=oxacillin, PEN=Penicillin, $\mathrm{GEN}=$ Gentamicin, ERY=Erythromycin, AMX=Amoxicillin, $\mathrm{LIN}=$ Lincomycin, $\mathrm{AMK}=$ Amikacin, $\mathrm{OFL}=$ Ofloxacin, $\mathrm{VAN}=$ Vancomycin

\subsection{Occurrence of MRSA with Respect to Demographics Factors}

The prevalence rates of MRSA according to age, gender, sample type and ward is shown on Table 1. MRSA isolation was more common in men and in individuals aged 21-40 years $(66.67 \%)$. Majority $(86.67 \%)$ of the MRSA were isolated from pus while no isolates were obtained from urine and throat swabs. Most (60\%) of the MRSA were isolated from patients in the surgical wards compared with other wards. However, these differences were not statistically significant $(\mathrm{P}>0.05)$.

Table 1. Distribution of MRSA isolates according to age, gender, type of sample and ward.

\begin{tabular}{lllll}
\hline Parameter & Category & Frequency & Percentage & P value \\
\hline \multirow{4}{*}{ Age (years) } & $\leq 20$ & 1 & 6.67 & \\
& $21-40$ & 10 & 66.67 & $\mathrm{P}=0.99$ \\
& $41-60$ & 2 & 13.33 & \\
Gender & Males & 2 & 13.33 & \\
& Females & 8 & 53.33 & $\mathrm{P}=0.65$ \\
& Pus & 13 & 46.67 & \\
Type of & Urine & 00 & 86.67 & \\
sample & Throat swab & 00 & 0.00 & \\
& Vaginal specimen & 1 & 0.00 & $\mathrm{P}=0.92$ \\
& Ear swab & 1 & 6.67 & \\
\multirow{4}{*}{ Hospital } & ICU & 1 & 6.67 & \\
ward & Surgical ward & 9 & 6.67 & \\
& General ward & 1 & 60.00 & \\
& Orthopaedic ward & 2 & 6.67 & $\mathrm{P}=0.63$ \\
& Outpatient & 2 & 13.33 & \\
\hline
\end{tabular}

\subsection{Distribution of MRSA with Respect to Some Predisposing Factors}

The highest MRSA isolates $66.7 \%(\mathrm{n}=10)$ were from patients with a hospital duration of more than 10 days $(\mathrm{P}=0.94)$ (Table 2). Participants with history of antibiotic use recorded the highest number of isolates for MRSA $100 \%$ $(n=15) \mathrm{P}=0.85$, individuals who used antibiotics frequently and those who used non prescribed antibiotics recorded highest number of MRSA isolates of $53.33 \%(n=8) \mathrm{P}=0.88$ and $86.7 \%(n=13) \mathrm{P}=0.92$ respectively. All the $\mathrm{p}$ values were statistically insignificant.

Table 2. Distribution of MRSA with respect to some predisposing factors.

\begin{tabular}{lllll}
\hline Parameter & Category & Frequency & Percentage & P value \\
\hline \multirow{4}{*}{$\begin{array}{l}\text { Duration of } \\
\text { hospitalisation }\end{array}$} & Less than 10 days & 3 & 13.33 & \\
& $\begin{array}{l}\text { More than } 10 \\
\text { days }\end{array}$ & 10 & 20.00 & $\mathrm{P}=0.94$ \\
Use of & Yes & 15 & 66.67 & \\
antibiotic & No & 0 & 100.00 & $\mathrm{P}=0.85$ \\
Frequency of & Very often & 8 & 0.00 & \\
antibiotic use & Rarely & 3 & 53.33 & \\
& Cannot tell & 4 & 20.00 & $\mathrm{P}=0.88$ \\
Type of & Prescribed & 2 & 26.67 & \\
antibiotic & Non prescribed & 13 & 13.33 & $\mathrm{P}=0.92$ \\
\hline
\end{tabular}

\section{Discussion}

The overall prevalence rate of Staphylococcus aureus was $28.95 \%$ which is commensurate to previous reports [13], but 
higher than the rates observed in India [14,15], Bangladesh [16], and Yaoundé [7]. In contrast, higher rates of prevalence were reported in Nigeria [17], Ethiopia [18, 19, 20, 21], Congo [22], and Iran [23, 24]. In the present study, the prevalence of MRSA was $13.16 \%$ while that of MSSA was $15.79 \%$. This is higher than the 5.26\% reported in Peshawar, Pakistan [25] and lower than those in other previous reports $[7,13,18,19,21]$. This variation in the prevalence of MRSA could be attributed to differences in sample size, sample type and the laboratory procedures employed. It could also be due to differences in the biological characteristics of the $S$. aureus strains, and/or infection control practices.

Although there was no significant difference in the isolation of MRSA with respect to gender, age, specimen type and ward, MRSA was isolated more from men (53.33\%) than females (46.67\%). This observation is in agreement with a previous study carried out in Peshawar, Pakistan [25, 26]. The high occurrence of MRSA in pus compared with other specimens has also been reported [27]. This is probably due to the fact that $S$. aureus is a common pathogen of the skin [2]; hence, more skin specimens were obtained.

In the present study, the highest resistance of MRSA isolates was observed against the penicillins. This is obvious as most Gram-positive bacteria have acquired the genes for beta lactamase production. However, resistance against amoxicillin was lower $(66.6 \%)$ compared with other penicillins (80-100\%). These findings are in agreement with reports in Sudan and Cameroon, respectively [1, 7]. No resistance was observed against lincomycin, amikacin and ofloxacin. Lincomycin and amikacin are drugs which are not commonly used but reserved for severe infections and infections caused by multidrug resistant pathogens. The fact that these drugs are not commonly used in our setup may explain the absence of resistance.

Resistance to fluoroquinolones is emerging among Grampositive bacteria and it is mediated by changes in the bacterial target enzymes DNA gyrase and topoisomerase IV, which reduce drug binding, and by action of efflux pumps on the bacterial membrane $[28,29,30]$. The zero resistance to ofloxacin observed in the present study could be due to the fact that these bacteria have not developed these mechanisms. Multidrug resistance was observed amongst MRSA isolates. Most isolates exhibited resistance to at least three antibiotics. This observation is consistent with previous findings in which MRSA showed multidrug resistance to at least two antibiotics [21], and the isolates presented with 18 different resistance patterns [7].

It was also noted from the present study that participants with longer hospital stay recorded a higher $(66.7 \%)$ prevalence of MRSA than outpatients (13.3\%). This difference may be due to sampling bias since fewer outpatients participated in this study. These findings are in accordance with similar findings in Scotland that recorded a higher prevalence for MRSA among individuals with prolonged hospital stay [31]. This can be attributed to the fact that longer hospital stay increase chances of coming in contact with different pathogens especially when hygiene and sanitation measures are not well implemented. Moreover, participants with history of antibiotic use, those who use antibiotics frequently and those who use non-prescribed antibiotics recorded a MRSA prevalence of $100 \%, 53.3 \%$ and $86.7 \%$ respectively. These findings correspond with findings in Scotland where participants with great antibiotic exposure and use of non-prescribed antibiotics recorded higher MRSA prevalence [32].

\section{Conclusion}

The prevalence of isolation of MRSA from clinical specimens in the present study was $13.16 \%$. Antimicrobial susceptibility testing revealed high resistance $(80-100 \%)$ to penicillins and no resistance to amikacin, lincomycin and ofloxacin. Multidrug resistance was also observed among MRSA isolates. There is need for the improvement of surveillance systems to monitor and curb the spread of resistant pathogens.

\section{References}

[1] S. I. Kheder, N. A. Ali, A. I. Fathelrahman. "Prevalence and Antimicrobial Susceptibility Pattern of Methicillin Resistance Staphylococcus in a Sudanese Surgical Ward" Pharmacology \& Pharmacy, 2012, 3, 103-108 http://dx.doi.org/10.4236/pp.2012.31015.

[2] L. K. Khanal, B. K. Jha. "Prevalence of methicillin resistant Staphylococcus aureus (MRSA) among skin infection cases at a hospital in Chitwan, Nepal" Nepal Med. Coll. J., 2010, 12, 224-228.

[3] World Health Organisation. The Burden of Health CareAssociated Infection Worldwide: A Summary. 2010. Accessed on July 19, 2016.

http://www.who.int/gpsc/country_work/summary_20100430_ en.pdf.

[4] J. Ojulong, T. P. Mwambu, M. Joloba, F. Bwanga, D. H. Kaddu-Mulindwa. "Relative prevalence of methicilline resistant Staphylococcus aureus and its susceptibility pattern in Mulago Hospital, Kampala, Uganda" Tanzan. J. Health Res, 2009, 11, 149-153.

[5] M. Cheesbrough. District Laboratory practice in tropical countries (Second Edition). Part 2. Page 45-143. New York: Cambridge University Press. 2006.

[6] C. E. Udobi, A. F. Obajuluwa, J. A. Onaolapo. "Prevalence and antibiotic resistance pattern of methicillin-resistant Staphylococcus aureus from an orthopaedic hospital in Nigeria" BioMed Res. Int. 2013, 860467.

[7] L. L. Njoungang, J. M. Nwobegahay, C. R. Ayangma, A. P. Njukeng, M. Kengne, E. M. Abeng, E. A. Mama, M. Tchouamo, D. T. Goon. "Prevalence and antibiotic resistance patterns of strains of Staphylococcus aureus isolated at the Yaoundé Military Hospital, Cameroon" Microbiol Res Int, 2015, 3(4), 56-63.

[8] C. E. Udobi, A. F. Obajuluwa, J. A. Onaolapo. "Prevalence and antibiotic resistance pattern of methicillin-resistant Staphylococcus aureus from an orthopaedic hospital in Nigeria" BioMed Res. Int. 2013, 860467. 
[9] B. Shrestha, B. Pokhrel T. Mohapatra. "Study of nosocomial isolates of Staphylococcus aureus with special reference to methicillin resistant $S$. aureus in a tertiary care hospital in Nepal" Nepal Med Coll J 2009, 11 (2), 123-126.

[10] C. Kesah, et al. "Prevalence of methicillin-resistant Staphylococcus aureus in eight African hospitals and Malta" Clin. Microbiol. Infect Off. Publ. Eur. Soc. Clin. Microbiol. Infect. Dis. 2003, 9, 153-156.

[11] A. B. Eyoh, M. Toukam, H. Gonsu, C. Fokunang, J. Atashili, E. E. Lyonga, G. Ikomey, B. Mukwele, M. Mesembe, N. Tsague, H. Mandi, M. C. O. Assoumou. "Antibiotics Susceptibility Profile of Staphylococcus aureus Isolated from the Anterior Nares of Hospital Personnel in Yaounde, Cameroon" Health Sci. Dis, 2013, 14 (2).

[12] Clinical and Laboratory Standards Institute (CLSI), Performance standards for antimicrobial susceptibility testing, Twenty-Fifth Informational Supplement, vol. 35, no. 3, 2015.

[13] J. Ojulong, T. P. Mwambu, M. Joloba, F. Bwanga, D. H. Kaddu-Mulindwa, "Relative prevalence of methicilline resistant Staphylococcus aureus and its susceptibility pattern in Mulago Hospital, Kampala, Uganda" Tanzan. J. Health Res. 2009, 11, 149-153.

[14] S. V. Kolhe, R. S. More, M. S. Mangalkar "Incidence of Antibiotic Resistance in Methicillin Resistant Staphylococcus aureus in Burn Patients in a Tertiary Care Hospital, India" Int J Pharm Bio Sci 2014, 5 (4): 537-541.

[15] Bilal Ahmad Mir, Dr. Srikanth. Prevalence and antimicrobial Susceptibility Of Methicillin Resistant Staphylococcus aureus and Coagulase-Negative Staphylococci in a Tertiary Care Hospital. Asian Journal Of Pharmaceutical And Clinical Research. Vol 6, Suppl 3, 2013. ISSN - 0974-2441.

[16] T. A. B. Islam, S. M. Shamsuzzaman "Prevalence and antimicrobial susceptibility pattern of methicillin-resistant, vancomycin-resistant, and Panton-Valentine leukocidin positive Staphylococcus aureus in a tertiary care hospital Dhaka, Bangladesh" Tzu Chi Medical Journal 2015, 27 (1), $10-14$.

[17] C. E. Udobi, A. F. Obajuluwa, J. A. Onaolapo. "Prevalence and antibiotic resistance pattern of methicillin-resistant Staphylococcus aureus from an orthopaedic hospital in Nigeria" BioMed Res. Int. 2013, 860467.

[18] T. Dilnessa, A. Bitew "Antimicrobial Susceptibility Pattern of Staphylococcus aureus with Emphasis on Methicillin Resistance with Patients Postoperative and Wound Infections at Yekatit 12 Hospital Medical College in Ethiopia" American Journal of Clinical and Experimental Medicine, 2016, 4 (1), 7 12 .

[19] Z. Taddesse, M. Tiruneh M. Gizachew "Staphylococcus aureus and its Antimicrobial Susceptibility Pattern in Patients, Nasalcarage of Health Personnel, and objects at Dessie referral hospital, Northern Ethiopia" Global Journal of Medical research: cMicrobiology and Pathology, 2014, 14 (2).
[20] T. Alebachew, G. Yismaw, A. Derabe, Z. Sisay "Staphylococcus aureus burn wound infection among patients attending yekatit 12 hospital burn unit, addis ababa, ethiopia" Ethiop. J. Health Sci 2012, 22, 209-213.

[21] A. Kahsay, A. Mihret, T. Abebe, T. Andualem "Isolation and antimicrobial susceptibility pattern of Staphylococcus aureus in patients with surgical site infection at Debre Markos Referral Hospital, Amhara Region, Ethiopia" Public Health Arch. Belg. Santé Publique 2014, 72, 16.

[22] J. M. L. Iyamba, J. M. Wambale, C. M. Lukukula, N. B. Takaisi-Kikuni "High prevalence of methicillin resistant staphylococci strains isolated from surgical site infections in Kinshasa" Pan Afr. Med. J. 2014, 18, 322.

[23] A. Ekrami, E. A. Montazeri, G. A. Kaydani, L. Shokoohizadeh "Methicillin Resistant Staphylococci: Prevalence and susceptibility patterns in a burn center in Ahvaz from 2013-2014, Iran" J. Microbiol, 2015, 7, 208-213.

[24] H. Momtaz, L. Hafezi "Methicillin-resistant Staphylococcus aureus isolated from Iranian hospitals: virulence factors and antibiotic resistance properties" Bosn. J. Basic Med. Sci, 2014, $14,219-226$

[25] R. A. Khan, A. U. Rahman, A. Ahmad, M. Jaseem, A Jabbar, Shad A. Khan, A. Iqbal, N. Ahmad, A. Wadood, T. U. Rahman "Prevalence and Antibiotic Susceptibility Profile of Methicillin-Resistant Staphylococcus aureus (MRSA) Isolated from Different Clinical Samples in District Peshawar" J. Appl. Environ. Biol. Sci., 2014, 4 (8S), 40-46.

[26] K. Mahmood, T. Tahir, T. Jameel, A. Ziauddin, H. F. Aslam "Incidence of Methicillin-resistant Staphylococcus aureus (MRSA) Causing Nosocomial Infection in a Tertiary Care Hospital" Annals, 2010, 16 (2), 91-96.

[27] H. K. Tiwari, A. K. Das, D. Sapkota, K. Sivrajan, V. K. Pahwa "Methicillin resistant Staphylococcus aureus: prevalence and antibiogram in a tertiary care hospital in western Nepal" J. Infect. Dev. Ctries 2009, 3, 681-684.

[28] L. J. V. Piddock "Fluoroquinolone resistance: Overuse of fluoroquinolones in human and veterinary medicine can breed resistance" BMJ 1998, 317, 1029-1030.

[29] G. A. Jacoby "Mechanisms of Resistance to Quinolones. Clin. Infect. Dis. 41, 2005, S120-S126.

[30] K. Poole "Efflux-Mediated Resistance to Fluoroquinolones in Gram-Positive Bacteria and the Mycobacteria" Antimicrob. Agents Chemother. 2000, 44, 2595-2599.

[31] S. Krishna, P. Divya, S. Shafiyabi "Post-operative surgical wound infections with special reference to methicillin resistant Staphylococcus aureus: an experience from Vims Hospital, Ballari. J. Biosci Tech, 2015, 6 (3), 697-702.

[32] K. C. Eseonu, S. D. Middleton, C. C. Eseonu "A retrospective study of risk factors for poor outcomes in methicillin-resistant Staphylococcus aureus (MRSA) infection in surgical patients" J. Orthop. Surg. 2011, 6, 25-25. 